\title{
Human Position/Height Detection Using Analog Type Pyroelectric Sensors
}

\author{
Shinya Okuda, Shigeo Kaneda, and Hirohide Haga \\ Graduate School of Engineering, Doshisha University, \\ 1-3 Tatara-Miyakodani, Kyotanabe City 610-0321, Japan \\ tam@ishss10.doshisha.ac.jp
}

\begin{abstract}
Pyroelectric sensors can detect changes of infrared rays. Usually, typical pyroelectric sensors are the digital output type, used for lighting switches and security systems. We can acquire, however, limited information from such digital on or off signals. We can also acquire a wealth of sensor data by using the analog type. Thus, this paper proposes a new approach to human position detection that discriminates human height (adults or children) by using analog type pyroelectric sensors. The proposed method employs Fast Fourier Transform (FFT) to calculate human position and analyzes the spectrum distribution to discriminate between adults or children. We built an experimental room 2.5 meters square and 2.5 meters high. Analog type sensors were installed at intervals of 0.8 meters in a grid shape. The proposed position detection method can calculate human position even if two persons are in the same room. Our height detection method that discriminates between adults and children is almost $90 \%$ accurate. Future research targets are improvements in accuracy and the development of an application system using these sensors.
\end{abstract}

\section{Introduction}

Pyroelectric sensors detect the feeble far-infrared rays emitted by the human body. They are usually used in lighting switches and security sensors. For instance, they are installed in rest room ceilings and passageways to save energy. These sensors have the following features: 1) can be used in a completely dark room without light; 2) no invasion of privacy problems; 3) nothing to be equipped for human body.

We arranged these sensors in the shape of a lattice on the ceiling and determined the position and height detection techniques from the data when examinees passed through their bottom. However, typical digital-type far-infrared sensors are immediately activated if a detected object enters detection range and this state continues for a fixed time. For this reason, the information from sensors is either on or off. We cannot detect when examinees passed directly under sensors.

Thus, we decided to treat the data of sensors as analog signals. In addition, we proposes timing detection techniques when passing the sensors and for height differences between adults and children to consider the input of context-aware services corresponding to adults and children. 

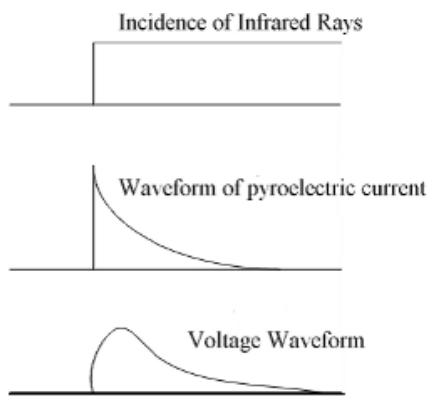

Fig. 1. Theoretical Waveforms
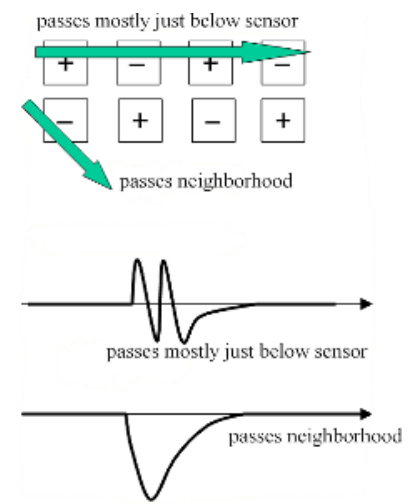

Fig. 2. Polar Characteristics of Detection Area and Output Waveforms

The remainder of this paper is organized as follows. In Section 2, we explain the waveforms of analog type pyroelectric sensors and in Section 3 propose detection techniques. In Section 4, we evaluate our experiments. Conclusions are drawn in Section 5.

\section{Output Waveforms of Pyroelectric Sensors}

Conventional position and height detection techniques use image processing, RFID (Radio Frequency Identification), ultra-sonic wave and etc. They can acquire fixed accuracy, but it is difficult to use them in everyday life. Image processing requires lighting system which is not available for sleeping time. RFID tag is far from handsfree. Ultra-sonic sensor requires endless ultra-sonic power emission. Since these demerits are practically serious obstacles, we used a different device and approach.

First, we focus the output waveforms of having one pyroelectric element. With a pyroelectric element, if the element's temperature rises by infrared rays, polarization will arise. Infrared rays are detected by locating the current produced when it is neutralized. Then the current is amplified with a DC amplifier with very high input impedance. Figure 1 shows the theoretical output signal waveform.

As shown in the upper part of Fig. 1, the amount of infrared rays that conduct incidence to an element increases. The current flowing from the pyroelectric elements is shown in the middle part of Fig. 1. Finally, the outputted signal is shown at the bottom. These sensors detect only changes in the amount of infrared rays; they can detect human movement, but not when the movement stops. The rising edge of output voltage is steep, and the output voltage tail is comparatively loose.

Below we consider a passing object as a shape of dot. Looking at the top of Fig. 2, "passes mostly just below a sensor" shows that a passing object goes through the plus and minus areas in sequence. First, the voltage swings to the 
plus direction, and then the passing object immediately moves to the next minus area. At this time such a phenomenon as the "signal will go up to positive, but flicks off in the opposite direction" occurs. By the same token, the infrared ray source moves to the next positive area and swings over into the positive direction again.

Finally, in cases of "passes mostly just below the sensor," the signal's characteristic is comparatively small amplitude, and the cycle is also short, except the tail, until the electric charge in an element is stabilized. (See the bottom of Fig. 2). The cycle depends on the source of the infrared passage speed. Frequency becomes high, so the movement of the object is quick, and amplitude becomes small. On the contrary, when it passes so that one domain may be grazed (passes the neighborhood in Fig. 2), the frequency ingredient is low, and amplitude increases. Consideration is summarized to below.

- Although the tail portion waveform has a long cycle and large amplitude, the object has already passed underneath a sensor by then.

- A high frequency ingredient does not appear if the infrared ray source does not pass through the center of the detection area. Therefore, if a high frequency ingredient is taken out, it is possible to capture the timing at which the infrared ray source has passed the detection range of a sensor.

\section{Proposal Techniques}

In this section, we describe the position detection and height difference detection techniques that used pyroelectric sensors. Moreover, these techniques depend on passage speed for output signals. Since, we compute passage speed between sensors, how to add this to the parameters of height difference detection is described.

\subsection{Position Detection Technique}

The sensors output signals after the infrared ray source has passed the detection area and until the electric charge in the elements are stabilized, as in Fig. 2. Since the portions of the last signals are low frequency waves, it is difficult to obtain the actual time of passing from raw waveforms. Furthermore, since the output value of a signal is also changed by passage speed, the temperature of the measurement environment, etc., the output maximum cannot simply be the recent side of a sensor. Time series detection of passing order is also difficult because part of the detection domain overlaps the contiguity sensors and output is mixed.

Since the waves of a low frequency ingredient are contained in the signals, the above problem is produced. Therefore, we developed the following procedures to detect human position. The processing outline is shown in Fig. 3.

\section{[Position Detection Technique Procedure]}

- STEP 1: Waveforms transform a time domain into a frequency domain using Fast Fourier Transform (FFT). 


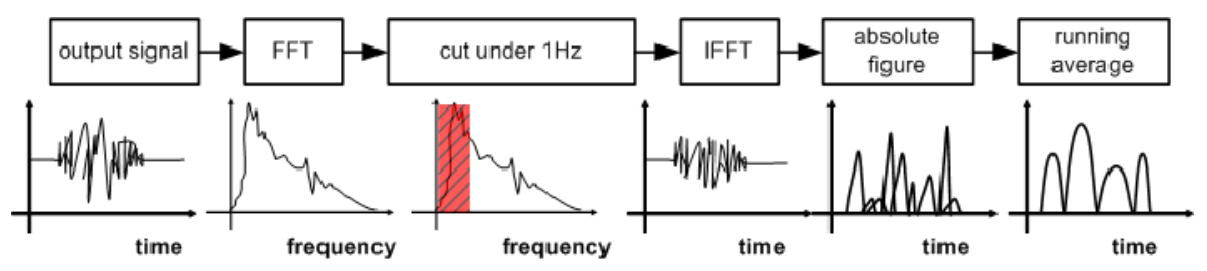

Fig. 3. Processing Procedure of Position Detection

- STEP 2: Cut the comparatively low frequency ingredient, which is set under $1 \mathrm{~Hz}$ in this evaluation.

- STEP 3: Transform a frequency domain into a time domain using Inverse Fourier Transform (IFFT)

- STEP 4: Calculate the running average every 150 data.

- STEP 5: Peaks are assessed as points of passing under the sensors.

The output signals of pyroelectric sensors are assumed to be unsaturated by this technique. Therefore, when there are two or more human beings, each effect will probably show up as output as an effect of liner superposition. On the contrary, such a frequency ingredient as "passes mostly just below sensors" appears. The span that appeared in high frequency is not passing. The infrared ray source "exists" in the detection domain of the sensors in that span. Even if the object has passed some areas at the edge of the entire detection domain, a peak appears in the timing. Moreover, the above argument is applicable in cases where an object passes through the domain of "+" and "-" in alternate shifts. Generally, it may pass through a domain aslant. In this case, the possibility that a complicated frequency ingredient will appear cannot be denied.

\subsection{Height Detection Technique}

In this section, we describe a height difference detection technique that measures the height differences of humans who enter detection areas and discriminates the height differences of children from adults (more than $170 \mathrm{~cm}$ and less than 100 $\mathrm{cm}$ ) considering the input as context-aware.

First, the relation between height and detected frequency is considered. A sensor has a detection range of two or more pluses and minus. Detection range becomes large when passing through the place distant from the sensor. When tall, the portion with a narrow detection area interval is passed, and when low, the portion into which the interval has spread is passed. (Refer to Fig. 4) As a result, when tall, the wave that contains a higher frequency ingredient than low cases is outputted.

The above point is considered theoretically. As shown in Fig. 5, sensors are installed in the ceiling and at points in the floor to establish the following values:

- Max $[\mathrm{m}]$ : distance from a ceiling to floor

- Inter $[\mathrm{m}]$ : distance between centers of the detection domain in the Max 



Fig. 4. Height Difference and Passage Fig. 5. Theoretic Model of Detection Area Position

$-L[\mathrm{~m}]$ : height of the human being detected

$-v[\mathrm{~m} / \mathrm{s}]$ : passage of time

At this time, we assume that infrared source is human's head. The distance (Distance $[\mathrm{m}]$ ) between the centers of the detection range of the height of the head is shown by Formula 1.

$$
\text { Distance }=\frac{\text { Inter } \times(\operatorname{Max}-L)}{\operatorname{Max}}[\mathrm{m}]
$$

One wavelength approximately corresponds to twice the distance of the centers of the detection range.

$$
F=\frac{v}{\lambda}=\frac{v}{2 \times \text { Distance }}[\mathrm{Hz}]
$$

Therefore, from the specification of sensor, when Max is $2.5 \mathrm{~m}$, Inter is about $0.4 \mathrm{~m}$. Frequency is $F=3.1 \mathrm{~Hz}$, if the height is $L=1.7 \mathrm{~m}$ when a man's walking speed is set to $v=0.8 \mathrm{~m} / \mathrm{s}$. On the other hand, if there is only $L=0.8 \mathrm{~m}$ height, frequency $F=0.92 \mathrm{~Hz}$ will become the highest frequency. The height difference turns into a frequency difference.

The power spectrum of frequency is compared with cases of being tall and short (expressed as "adult" and a "child" below for convenience.) In the former, an amplitude of $1 \sim 3 \mathrm{~Hz}$ is strong, and in the latter, ingredients of over $1 \mathrm{~Hz}$ are seldom included and the amplitude is considered small. Furthermore, amplitude is more often low for adults, on the whole, than for children's cases.

Thus, when signal waveform is transformed into a frequency domain using FFT, height differences notably appear as the difference of included frequency ingredient. The domain of characteristic frequency is established on the basis of the highest frequency $F \mathrm{~Hz}$ (Formula 2).

As mentioned above, the discrimination of adults and children is possible by comparing the power spectrum of a characteristic frequency domain. Actually, it processes stepping on the following procedure. 


\section{[Height Detection Technique Procedure]}

- STEP 1: Process FFT, about the data which human passed under the three sensors.

- STEP 2: Calculate the sum total value of the power in the three sensors.

- STEP 3: A low frequency domain is set to Field I and a high frequency domain is set to Field II, as sum total values.

- STEP 4: Calculate the average value of the power in Field I and Field II.

- STEP 5: Calculate the rate of Field II on the basis of Field I. This value is set to the Ratio.

- STEP 6: Judge as an adult or child if the Ratio is beyond or below the threshold.

Output waveform has considerable variation according to the measurement environment. So, it is not commonly understood that the absolute value of amplitude applies to appraisal standards. To solve this problem, we adopt Ratio as a relative value, which is calculated as follows.

$$
\text { Ratio }=\frac{\text { average }(\text { Field } \mathrm{I})}{\operatorname{average}(\text { Field } \mathrm{I})}
$$

\subsection{Speed Detection Technique}

The height difference detection technique mentioned above calculated a man's passage speed as about $0.8 \mathrm{~m} / \mathrm{s}$ and computed the Ratio value. Although $0.8 \mathrm{~m} / \mathrm{s}$ was said to be general walking speed, we also considered the detection of slower speeds $(v=0.5 \mathrm{~m} / \mathrm{s})$. Since the speed is slowed down, the range in which the features of an adult and a child appear in a power spectrum is basically shifted to a low frequency domain. Concrete passage speed calculation is performed in the following order.

\section{[Speed Detection Technique Procedure]}

- STEP 1: The time when the peak value of the beginning at the time of sensor domain penetration appears is detected in three sensors.

- STEP 2: Calculate the time passage between the first and second sensors and between the second and third.

- STEP 3: Passage speed is calculated from installation distance and time passage of sensors.

- STEP 4: The average value of the speed of two passages is $v \mathrm{~m} / \mathrm{s}$.

The height difference judging technique in which passage speed was also considered is described as follows. If $v \mathrm{~m} / \mathrm{s}$ is beyond the threshold, it is set to "normal." If not, it is set to "slow." Then an applicable Field is set, and height difference is judged. 


\section{Evaluations by Experiments}

\subsection{Experimental Equipment}

Figure 6 shows a sensor circuit that we built as a prototype. We installed nine circuits in the shape of a lattice at intervals of $0.8 \mathrm{~m}$ and heights of $2.5 \mathrm{~m}$. All equipment is shown in Fig. 7. The sensor portion is analog output and spot detection type NaPiOn (Model Number: AMN23112) by Matsushita Electric Works, Ltd.

Normal NaPiOn sensors are too sensitive for correct measurements since the signal is saturated. It's difficult to analyze frequency from saturated signals. To attenuate infrared rays, a polyethylene board was placed in front of a sensor. When installation interval was $0.8 \mathrm{~m}$, overlap to the next sensor was large. To narrow the detection area, tape was stuck to the lens. Ideally, it should become the detection range shown in Fig. 8.

Sensor output is transmitted to computer by balanced transmission through a multicore shielded cable to avoid external noise influence at the time of signal transmission. An AD conversion board (from Interface Corporation, Japan) receives from the $\mathrm{PC}$ side. Sampling rate is $100 \mathrm{~Hz}$.



Fig. 6. Circuit

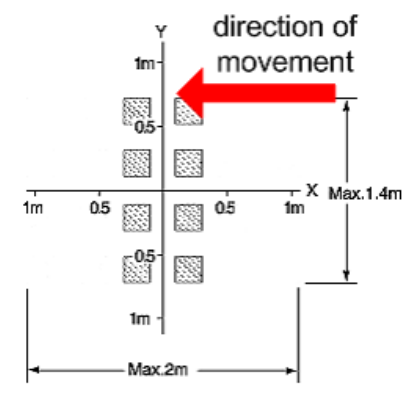

Fig. 8. Detection Range after Adjustment

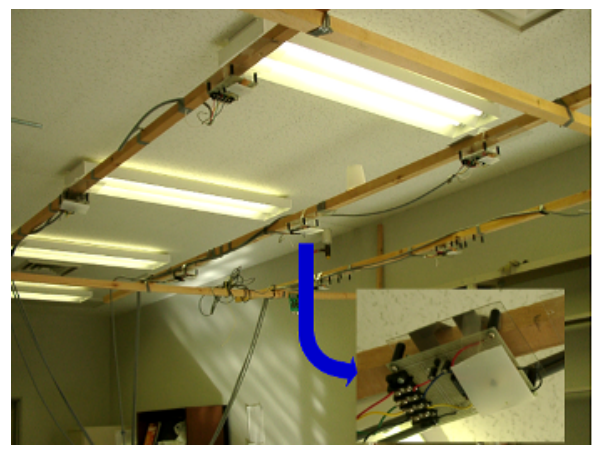

Fig. 7. Prototype System

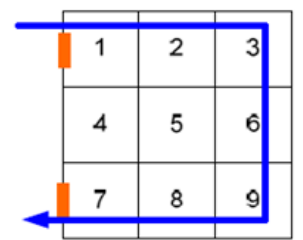

Fig. 9. Route for an Experiment 


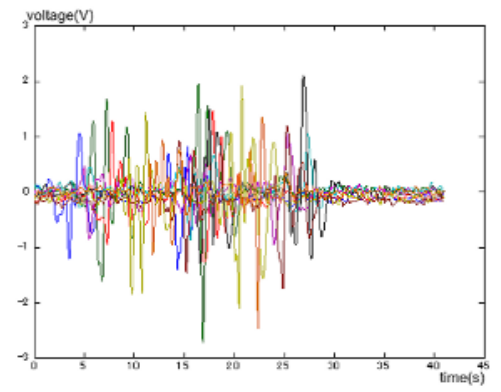

Fig. 10. Output Waveforms

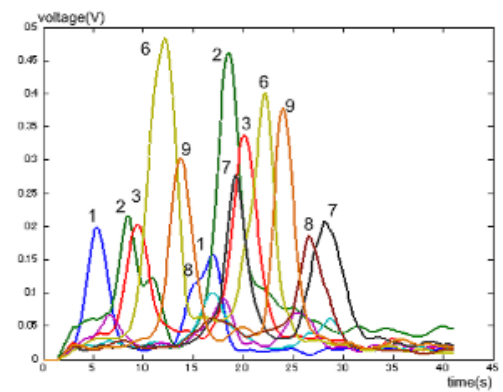

Fig. 11. Processed Waveforms

\subsection{Position Detection}

The installed sensors were numbered, as shown in Fig. 9. We conducted experiments in which two people passed detection areas in the following order: $1 \rightarrow 2$ $\rightarrow 3 \rightarrow 6 \rightarrow 9 \rightarrow 8 \rightarrow 7$; when the first person enters area 9 , the second person goes into area 1 . Walking speed is $0.5 \mathrm{~m} / \mathrm{s}$. And output waveforms are processed using the position detection technique shown in Section 3.1.

Sensor output waveform is shown in Fig. 10, and processing results are shown in Fig. 11. If a peak position is seen, it has passed in order. However, if the peak appeared at the same time as the sensor separated in position, distinguishing two or more individuals is possible.

\subsection{Height Detection}

We conducted position detection experiments using sensors 7, 8, and 9. The person passes under the sensor in a straight line. At this time, speed and height were changed and the following data number patterns were extracted. ("Adult" represents state of standing. "Child" represents state of crouching down)

- Adult - Normal Speed $(0.8 \sim 1.0 \mathrm{~m} / \mathrm{s})$

- Adult - Slow Speed $(0.3 \sim 0.5 \mathrm{~m} / \mathrm{s})$

- Child - Normal Speed $(0.8 \sim 1.0 \mathrm{~m} / \mathrm{s})$

- Child - Slow Speed $(0.3 \sim 0.5 \mathrm{~m} / \mathrm{s})$

We investigated the accuracy of distinguishing the above pattern by the proposed technique. For example, power spectrums are shown in which the adult and the child are in normal speed. (Figs. 12 and 13) Power spectrums of slow speed are shown in Figs. 14 and 15.

Comparisons of the power spectrums of the four patterns showed the following features. In cases of normal speed, a power of $2 \sim 3 \mathrm{~Hz}$ exists to some extent in the adult figures. On the other hand, such figures are very low in the data for children. In cases of slow speed, in the figures of both adults and children, the high power domain is generally low. As mentioned above, the range of Field I and II was set up as follows. 

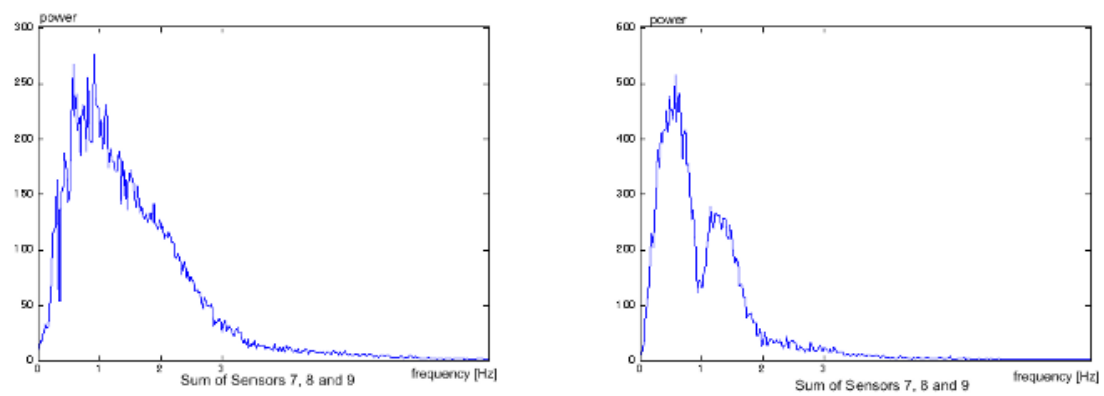

Fig. 12. Power Spectrum of Adult - Normal Fig. 13. Power Spectrum of Child - Normal Speed Speed
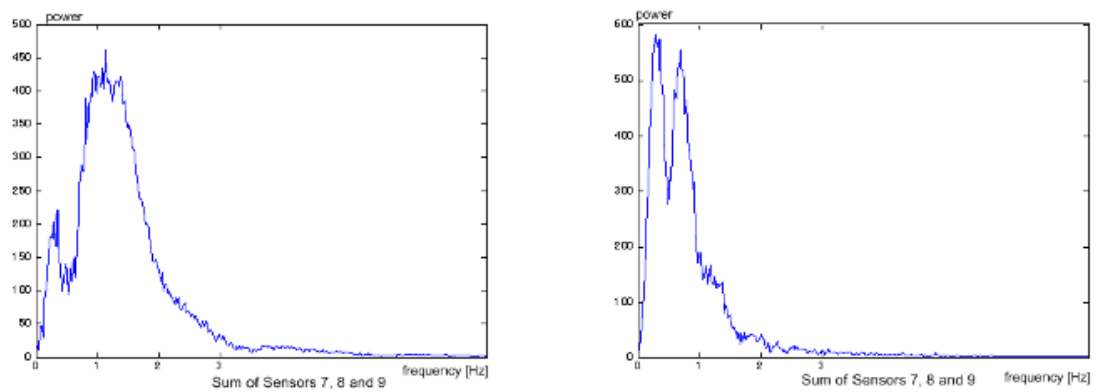

Fig. 14. Power Spectrum of Adult - Normal Fig. 15. Power Spectrum of Child - Normal Speed Speed

- Normal Speed: Field I: $0 \sim 1 \mathrm{~Hz}$, Field II: $2 \sim 3 \mathrm{~Hz}$

- Slow Speed: Field I: $0 \sim 1 \mathrm{~Hz}$, Field II: $1 \sim 2 \mathrm{~Hz}$

We performed evaluations that checked the accuracy of the proposed techniques using the examinees of Table 1 . Results are shown in Table 2. The probability of making mistakes in height difference judgments is low, even when making mistakes in speed judging. Accuracy is improved by changing the do-

Table 1. Height of Examinees

Table 2. Evaluation Results

\begin{tabular}{|c|c|c|}
\hline Examinee & Standing & Crouching \\
\hline ex.1-male & 174 & 114 \\
\hline ex.2-male & 180 & 123 \\
\hline ex.3-male & 168 & 95 \\
\hline ex.4-male & 182 & 113 \\
\hline ex.5-male & 172 & 115 \\
\hline ex.6-female & 165 & 110 \\
\hline ex.7-female & 162 & 110 \\
\hline
\end{tabular}

\begin{tabular}{|c|c|c|}
\hline & Speed Mistakes & Height Mistakes \\
\hline Adult - Normal & $1 / 8$ & $0 / 8$ \\
\hline Child - Normal & $3 / 8$ & $1 / 8$ \\
\hline Adult - Slow & $3 / 8$ & $2 / 8$ \\
\hline Child - Slow & $1 / 8$ & $0 / 8$ \\
\hline Total & $8 / 32$ & $3 / 32$ \\
\hline Accuracy & $75 \%$ & $91 \%$ \\
\hline
\end{tabular}


main of Field I or II after speed judging. Therefore, even if it made a mistake in speed judging, it turned out that the judgment of height difference itself is seldom affected. The accuracy of height difference detection was realized about $90 \%$ of the time.

\section{Conclusions}

In this paper, we proposed a technique for other uses of pyroelectric sensors than a switch. Such sensors have the following features: cheap, no worries about privacy or generation of electric waves.

When this sensor is installed in a ceiling in the shape of a lattice, the following phenomena can be detected. 1) Detection in the radius of about $0.8 \mathrm{~m}$ is possible in the position of the human being in the room. 2) The position detection of two people is also possible to some extent. 3) For children and adults in whom height greatly differs, detection (adults or children) is possible.

Since this sensor does not have such a quick response speed, it is difficult to use when we need high accuracy. Our future targets include more accurate detection techniques to take advantage of this sensor's features. A concrete service proposal is determined and cooperation with other sensors is sought.

\section{References}

[1] Shinya Tamano, Ryusuke Nakatani, Shigeo Kaneda, and Hirohide Haga: Position discernment technique using multi pyroelectric sensors. 66th IPSJ, 4H-7, March, 2004, In Japanese.

[2] Kazutaka Okamoto: Pyroelectric sensors with a self-diagnostic function HORIBA Technical Report, No. 11, Sept., 1995. In Japanese.

[3] Koichi Matsumoto and Kazutaka Okamoto: Pyroelectric Sensors HORIBA Technical Report, No. 7, July 1993. In Japanese. 